\title{
Retraction
}

\section{Retracted: Partial Sums of Functions of Bounded Turning}

\section{International Journal of Mathematics and Mathematical Sciences}

Received 16 March 2014; Accepted 16 March 2014; Published 3 April 2014

Copyright (C) 2014 International Journal of Mathematics and Mathematical Sciences. This is an open access article distributed under the Creative Commons Attribution License, which permits unrestricted use, distribution, and reproduction in any medium, provided the original work is properly cited.

This article has been retracted as it is essentially identical in title and technical content with a previously published paper in Journal of Inequalities in Pure and Applied Mathematics (JIPAM). The earlier publication is "Partial Sums of Functions of Bounded Turning” [1] Volume 4, Issue 4, Article 79, 2003.

\section{References}

[1] J. M. Jahangiri and K. Farahmand, "Partial sums of functions of bounded turning," International Journal of Mathematics and Mathematical Sciences, vol. 2004, no. 1, pp. 45-47, 2004. 
IJMMS 2004:1, 45-47

PII. S0161171204305284

http://ijmms.hindawi.com

(c) Hindawi Publishing Corp.

\section{PARTIAL SUMS OF FUNCTIONS OF BOUNDED TURNING}

\section{JAY M. JAHANGIRI and K. FARAHMAND}

Received 26 May 2003

We determine conditions under which the partial sums of the Libera integral operator of functions of bounded turning are also of bounded turning.

2000 Mathematics Subject Classification: 30C45, 26D05.

1. Introduction. Let $\mathscr{A}$ denote the family of functions $f$ which are analytic in the open unit disk $U=\{z:|z|<1\}$ and are normalized by

$$
f(z)=z+\sum_{k=2}^{\infty} a_{k} z^{k}, \quad z \in u \text {. }
$$

For $0 \leq \alpha<1$, let $\mathscr{B}(\alpha)$ denote the class of functions $f$ of the form (1.1) so that $\mathfrak{R}\left(f^{\prime}\right)>\alpha$ in $U$. The functions in $\mathscr{B}(\alpha)$ are called functions of bounded turning (cf. [4]). By the Nashiro-Warschowski theorem (see, e.g., [3]), the functions in $\mathscr{B}(\alpha)$ are univalent and also close-to-convex in $u$.

For $f$ of the form (1.1), the Libera integral operator $F$ is given by

$$
F(z)=\frac{2}{z} \int_{0}^{z} f(\zeta) d \zeta=z+\sum_{k=2}^{\infty} \frac{2}{k+1} a_{k} z^{k}
$$

The $n$th partial sums $F_{n}(z)$ of the Libera integral operator $F(z)$ are given by

$$
F_{n}(z)=z+\sum_{k=2}^{n} \frac{2}{k+1} a_{k} z^{k}
$$

In [6] it was shown that if $f \in \mathscr{A}$ is starlike of order $\alpha, \alpha=0.294, \ldots$, so is the Libera integral operator $F$. We also know that (see, e.g., [1]) there are functions which are univalent or spiral-like in $U$ so that their Libera integral operators are not univalent or spiral-like in $U$. Li and Owa [5] proved that if $f \in \mathscr{A}$ is univalent in $U$, then $F_{n}(z)$ is starlike in $|z|<3 / 8$. The number $3 / 8$ is sharp. In this note we make use of a result of Gasper [2] to provide a simple proof for the following theorem.

MAIN THEOREM. If $1 / 4 \leq \alpha<1$ and $f \in \mathscr{B}(\alpha)$, then $F_{n} \in \mathscr{B}((4 \alpha-1) / 3)$.

2. Preliminary lemmas. To prove our Main theorem, we will need the following three lemmas. The first lemma is due to Gasper (see [2, Theorem 1]) and the third lemma 
is a well-known and celebrated result (cf. [3]) that can be derived from the Herglotz' representation for positive real part functions.

LEMMA 2.1. Let $\theta$ be a real number and let $m$ and $k$ be natural numbers. Then

$$
\frac{1}{3}+\sum_{k=1}^{m} \frac{\cos (k \theta)}{k+2} \geq 0
$$

LEMMA 2.2. For $z \in U$,

$$
\mathfrak{R}\left(\sum_{k=1}^{m} \frac{z^{k}}{k+2}\right)>-\frac{1}{3}
$$

Proof. For $0 \leq r<1$ and for $0 \leq|\theta| \leq \pi$, write $z=r e^{i \theta}=r(\cos (\theta)+i \sin (\theta))$. By DeMoivre's law and the minimum principle for harmonic functions, we have

$$
\mathfrak{R}\left(\sum_{k=1}^{m} \frac{z^{k}}{k+2}\right)=\sum_{k=1}^{m} \frac{r^{k} \cos (k \theta)}{k+2}>\sum_{k=1}^{m} \frac{\cos (k \theta)}{k+2} .
$$

Now by Abel's lemma (cf. Titchmarsh [7]) and condition (2.1) of Lemma 2.1 we conclude that the right-hand side of (2.3) is greater than or equal to $-1 / 3$.

LEMmA 2.3. Let $P(z)$ be analytic in $U, P(0)=1$ and let $\mathfrak{R}(P(z))>1 / 2$ in $U$. For functions $Q$ analytic in $U$, the convolution function $P * Q$ takes values in the convex hull of the image on $U$ under $Q$.

The operator " $*$ " stands for the Hadamard product or convolution of two power series $f(z)=\sum_{k=1}^{\infty} a_{k} z^{k}$ and $g(z)=\sum_{k=1}^{\infty} b_{k} z^{k}$ denoted by $(f * g)(z)=\sum_{k=1}^{\infty} a_{k} b_{k} z^{k}$.

3. Proof of Main theorem. Let $f$ be of the form (1.1) and belong to $\mathscr{B}(\alpha)$ for $1 / 4 \leq$ $\alpha<1$. Since $\Re\left(f^{\prime}(z)\right)>\alpha$, we have

$$
\mathfrak{R}\left(1+\frac{1}{2(1-\alpha)} \sum_{k=2}^{\infty} k a_{k} z^{k-1}\right)>\frac{1}{2}
$$

Applying the convolution properties of power series to $F_{n}^{\prime}(z)$, we may write

$$
\begin{aligned}
F_{n}^{\prime}(z) & =1+\sum_{k=2}^{n} \frac{2 k}{k+1} a_{k} z^{k-1} \\
& =\left(1+\frac{1}{2(1-\alpha)} \sum_{k=2}^{\infty} k a_{k} z^{k-1}\right) *\left(1+(1-\alpha) \sum_{k=2}^{n} \frac{4}{k+1} z^{k-1}\right) \\
& =P(z) * Q(z) .
\end{aligned}
$$

From Lemma 2.2 for $m=n-1$, we obtain

$$
\mathfrak{R}\left(\sum_{k=2}^{n} \frac{z^{k-1}}{k+1}\right)>-\frac{1}{3}
$$


Applying a simple algebra to inequality (3.3) and $Q(z)$ in (3.2) yields

$$
\mathfrak{R}(Q(z))=\mathfrak{R}\left(1+(1-\alpha) \sum_{k=2}^{n} \frac{4}{k+1} z^{k-1}\right)>\frac{4 \alpha-1}{3} .
$$

On the other hand, the power series $P(z)$ in (3.2) in conjunction with the condition (3.1) yield $\mathfrak{R}(P(z))>1 / 2$. Therefore, by Lemma $2.3, \mathfrak{R}\left(F_{n}^{\prime}(z)\right)>(4 \alpha-1) / 3$. This concludes the Main theorem.

REMARK 3.1. The Main theorem also holds for $\alpha<1 / 4$. We also note that $\mathscr{B}(\alpha)$ for $\alpha<0$ is no longer a bounded turning family.

\section{REFERENCES}

[1] D. M. Campbell and V. Singh, Valence properties of the solution of a differential equation, Pacific J. Math. 84 (1979), no. 1, 29-33.

[2] G. Gasper, Nonnegative sums of cosine, ultraspherical and Jacobi polynomials, J. Math. Anal. Appl. 26 (1969), 60-68.

[3] A. W. Goodman, Univalent Functions. Vol. I, Mariner Publishing, Florida, 1983.

[4] _ Univalent Functions. Vol. II, Mariner Publishing, Florida, 1983.

[5] J.-L. Li and S. Owa, On partial sums of the Libera integral operator, J. Math. Anal. Appl. 213 (1997), no. 2, 444-454.

[6] P. T. Mocanu, M. O. Reade, and D. Ripianu, The order of starlikeness of a Libera integral operator, Mathematica (Cluj) 19(42) (1977), no. 1, 67-73.

[7] E. C. Titchmarsh, The Theory of Functions, 2nd ed., Oxford University Press, London, 1975.

Jay M. Jahangiri: Department of Mathematics, Kent State University, Burton, Ohio 44021-9500, USA

E-mail address: jay@geauga.kent.edu

K. Farahmand: School of Computing and Mathematics, University of Ulster, Jordanstown Campus, Newtownabbey Co. Antrim BT37 0QB, UK

E-mail address: k.farahmand@uTster.ac.uk 